\title{
Stellar map centroid positioning based on dark channel denoising and feasibility of jitter detection on ZiYuan3 satellite platform
}

\author{
Hong Zhu, ${ }^{a}$ Junfeng Xie,, ,* Xinming Tang, ${ }^{b}$ Di Jia, ${ }^{c}$ and Guangtong Sun ${ }^{a}$ \\ anstitute of Disaster Prevention, College of Ecology and Environment, Beijing, China \\ ${ }^{b}$ Land Satellite Remote Sensing Application Center, Ministry of Natural Resources, \\ Beijing, China \\ ${ }^{c}$ Liaoning Technical University, School of Electronic and Information Engineering, \\ Huludao, China
}

\begin{abstract}
Stellar map denoising and centroid positioning, which directly determine the postpositioning accuracy of star trackers, are key technologies in stellar map processing. Due to the influence of a complex starry sky background, there is often a large amount of noise in stellar maps, which makes it difficult to accurately locate the stellar centroid. A stellar map processing method based on dark channel denoising and continuous multiframe stellar map centroid positioning combined with centroid trajectory constraints is proposed. First, a dark channel noise template is used for denoising, and the single-point and multipoint noises in the denoising result are filtered. Second, in the process of stellar map positioning, if the maximum gray value of the stellar is not unique, it is constrained by the previous stellar positioning result, an adaptive window is established, and the gray-scale centroid weighting method is used to locate the centroid. Then, the star angular distance is used to analyze the precision of the centroid positioning. Finally, the jitter frequency of the satellite platform based on the continuous multiframe centroid positioning result is used to detect satellite attitude. The experimental results show that the performance of dark channel denoising, which can solve many strip noise and background noise problems in stellar maps, is better than that of the existing stellar map denoising method. The centroid positioning results improves the star angular distance by 18.85 arc sec compared with the Gaussian filter and by 8.03 arc sec compared with the global threshold segmentation method, significantly improving the accuracy of stellar map centroid positioning and laying a foundation for improving the star tracker positioning accuracy. A jitter frequency of $0.67 \mathrm{~Hz}$ was detected on the ZiYuan3 satellite platform based on the centroid positioning results. It enriches the jitter detection methods and provides a theoretical and technical basis for the design of future high-resolution ground observation remote sensing satellite platforms and geometric accuracy compensation. () The Authors. Published by SPIE under a Creative Commons Attribution 4.0 Unported License. Distribution or reproduction of this work in whole or in part requires full attribution of the original publication, including its DOI. [DOI: 10.1117/1.JRS.15.016519]
\end{abstract}

Keywords: Stellar map; centroid positioning; dark channel denoising; jitter detection; star angular distance.

Paper 200466 received Jun. 22, 2020; accepted for publication Nov. 23, 2020; published online Mar. 12, 2021.

\section{Introduction}

Star trackers have the advantages of high precision, good reliability, strong acquisition, and tracking ability, which are key factors in determining whether a satellite can operate stably in orbit. ${ }^{1-3}$ The measurement accuracy of the star trackers has a great influence on the pointing accuracy and attitude stability of the satellite platform. The pointing accuracy and attitude stability of the satellite platform directly determine the uncontrolled mapping capability of the remote sensing satellite. In the star tracker attitude control system, the star trackers determine the orientation of the star tracker camera axis in the celestial coordinate system through a series

*Address all correspondence to Junfeng Xie, xiejf@lasac.cn.com 
of tasks such as stellar map shooting, centroid positioning, stellar map recognition, and attitude calculation, to calculate the attitude of the spacecraft. ${ }^{4,5}$ As one of the main data transmitted by the star trackers, stellar map can assist the postattitude calibration through stellar map recognition and matching. Moreover, stellar map denoising and centroid positioning are the significant technologies of star tracker attitude determination. The complex space environment leads to a large amount of nonuniform noise in the stellar map shot by the star trackers. Stellar map denoising is the premise of centroid positioning and the results directly determine the postattitude determination of the satellite sensors. ${ }^{6-8}$ Therefore, effectively mitigating noise and accurate centroid positioning have become one of the important research areas of aerospace remote sensing satellites in recent years. ${ }^{9,10}$

Currently, stellar map denoising methods can be roughly divided into two categories: stellar map denoising based on filtering or threshold segmentation method. Stellar maps based on filtering denoising ${ }^{8,11}$ mainly include mean filtering, median filtering, Gaussian low-pass filtering, and Wiener filtering. Filtering denoising methods can mitigate the speckle noise in natural images better. However, the targets in the stellar maps are distributed in a spot-like pattern, which is similar to the noise distribution. ${ }^{12}$ The stellar information is missing in the process of the denoising based on filtering denoising methods. Obviously, these methods are applied to the noise mitigation of stellar maps and they have great limitations. The threshold segmentation method $^{13}$ mitigates the noise in the stellar map through threshold constraints. Usually, the average value of the energy of each pixel in the stellar map and the median error of $n$ times are used as the global threshold ( $n$ is generally 3 to 5). The edge of the stellar map obtained by the ZiYuan3 (ZY-3) satellite platform is strongly exposed, and it carries obvious band noise, as shown in Fig. 1. At present, there is no global threshold suitable for all stellar maps. Thus, threshold segmentation method is difficult to mitigate the complex noise carried by the stellar maps. Then how to mitigate the edge band noise in the stellar map while improving the processing efficiency is an urgent problem to be solved in the postattitude of the satellite platform attitude.

In the field of centroid positioning, experts and scholars have also carried out much research and have achieved certain research results. Centroid positioning methods mainly include grayscale weighting, ${ }^{14}$ Gaussian surface fitting, ${ }^{15,16}$ and ellipse fitting. ${ }^{17}$ The relationship between pixel values and weights for centroid positioning is considered in the gray-scale weighting method. These methods are relatively simple. The Gaussian function is used in the Gaussian surface fitting method to simulate the point spread function in the imaging process, which is used to locate the centroid of the stellar field. The stability is good, but the calculation is relatively complicated. In the ellipse fitting method, the stellar field is treated as an ellipse. The morphology is adopted to extract the edge of the stellar field, and then to locate the centroid through the least squares fitting. Unfortunately, this method is not stable. Motivated by this, how to improve the method of centroid positioning is very significant. From the perspective of attitude data processing, improving the denoising results of the stellar map, improving the centroid positioning accuracy, and creating favorable conditions for the postattitude processing of the satellite platform will have important theoretical significance and application value for improving the geometric positioning accuracy of satellite images.

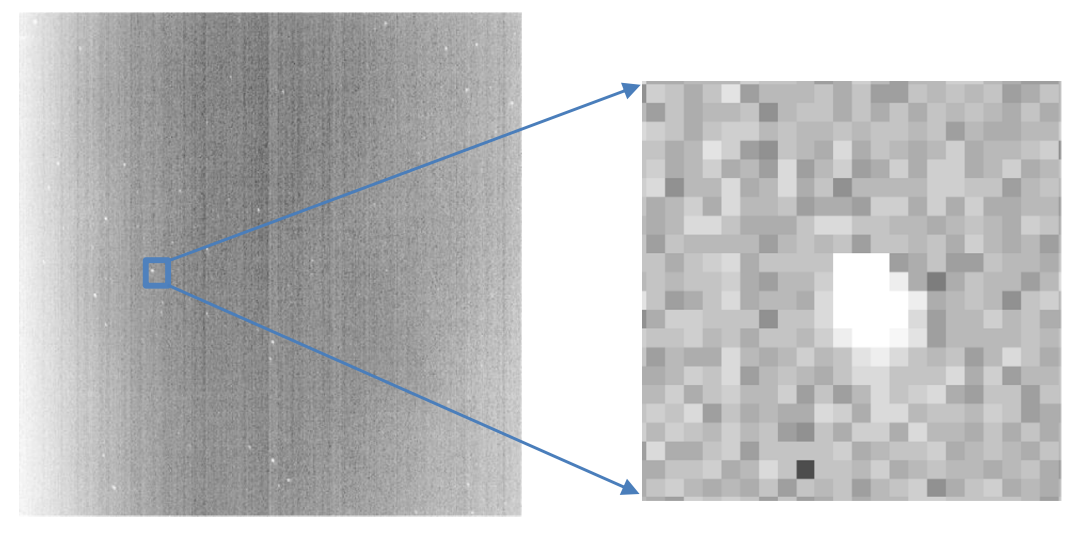

Fig. 1 Original stellar map. 
ZY-3 is China's first civilian high-resolution stereo surveying and mapping satellite. In terms of attitude equipment, the ZY-3 satellite platform carries three star trackers and four gyro instruments. ${ }^{18-20}$ The star trackers and the gyros jointly integrate the attitude determination system. In order to further improve the postattitude processing accuracy of the ZY-3 satellite platform, the stellar map obtained by the star tracker, which is installed on the ZY-3 satellite platform, is studied in this paper. The problems of stellar map denoising and centroid positioning accuracy are mainly solved. Meanwhile, the feasibility of the centroid change in the continuous multi-frame stellar map for the jitter detection of satellite platform is further analyzed. These works will accumulate a certain technical reserve for the subsequent mitigation of attitude jitter and posture accuracy improvement.

\section{Materials and Methods}

For the stellar map obtained from ZY-3, the stellar map noise information mainly includes system background noise and random noise. Given the noise characteristics of stellar maps and stellar positioning accuracy, stellar map denoising based on dark channel and the continuous multiframe stellar map centroid positioning combined with centroid trajectory constraints is put forward in this paper. Finally, the jitter frequency of the satellite platform based on the continuous multiframe centroid positioning results is analyzed. Figure 2 illustrates the overall flowchart of the method in this paper.

\subsection{Stellar Map Denoising Based on Dark Channel}

The noise of stellar maps to have a certain correlation and weak difference due to the impact of space environment changes, hardware, and current.

The special integrated structure and external environment of the active pixel sensor (APS) star tracker show obvious strip noise and a large amount of nonuniform noise in the vertical direction of the stellar map. This noise can be considered systematic background noise because of the slight variations in continuous multiframe stellar maps. Moreover, regardless of the band noise, the system background noise is much smaller than the pixel gray value of the stellar field. Consequently, the dark channel noise template of the APS stellar map was extracted according to the minimum pixel value of the sequence stellar map. Then the noise information in the stellar map will be greatly mitigated. For an arbitrary stellar map J, the mathematical description of the dark channel noise template extraction is given as

$$
I^{\text {noise }}(x)=\min _{y \in \Omega(x)}\left[\min _{c \in\left\{J^{1}, J^{2}, \ldots, J^{n}\right\}} J^{c}(y)\right], \quad \text { where } c=\left\lceil\frac{R \_ \text {star }}{S / T} / t\right\rceil,
$$

where $I^{\text {noise }}$ represents the extracted dark channel noise template information, $J^{c}$ represents the stellar map sequence, $\Omega(x)$ represents a window centered on pixel $x, R \_$star is the diameter of the

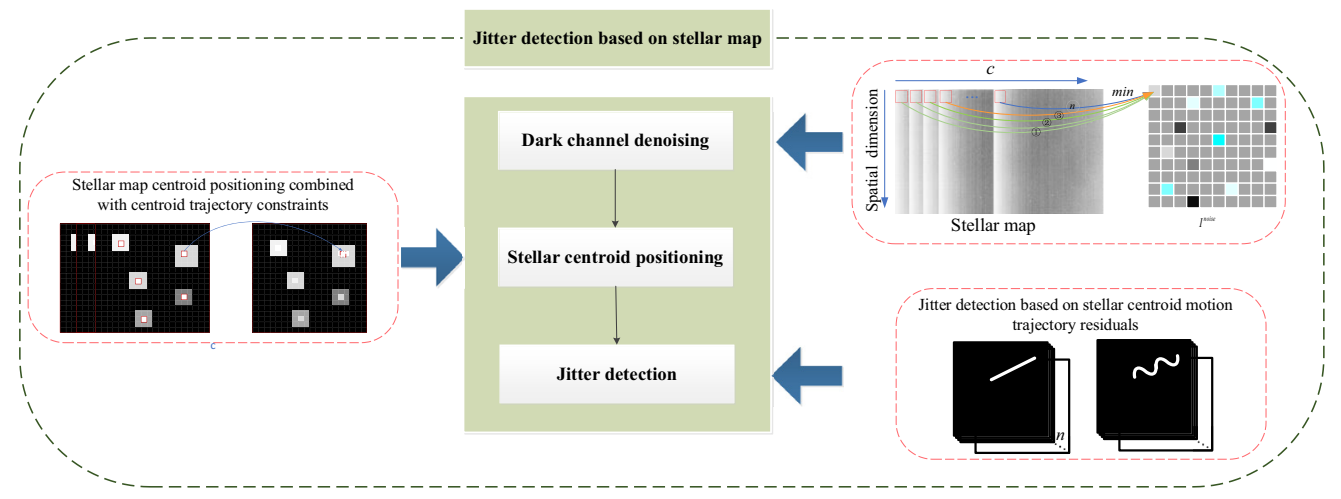

Fig. 2 The overall technical flowchart of this paper. 


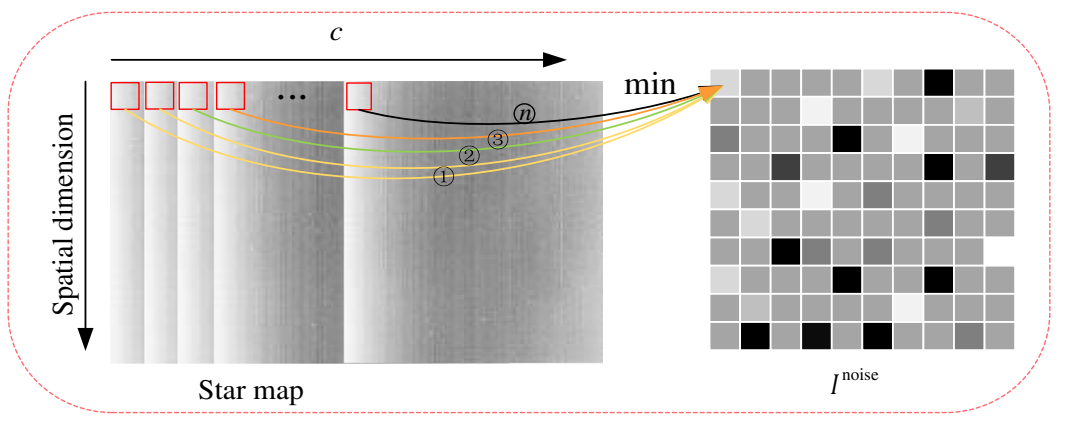

Fig. 3 Schematic diagram for calculating stellar map noise.

star, $t$ is the stellar map exposure time, $S$ is the displacement of the star, $T$ is the accumulated time within displacement $S$, and $\lceil\cdot\rceil$ represents rounding up.

As shown in Fig. 3, the number $c$ of stellar map frames exposed in time $T$ that is required for a star spot to move out of a stellar map was calculated. The dark channel noise template of the sequence stellar maps was obtained by calculating the minimum value of each pixel of the $c$ frame stellar map. The difference between the sequence stellar map and the dark channel noise template was determined to mitigate the noise. Then the continuous multiframe stellar maps are denoised.

The stellar maps based on dark channel denoising remain as single-point or multipoint noise. This noise consumes storage resources and affects the efficiency and accuracy of centroid positioning. In order to accurately locate the stellar centroid, the residual noise of the stellar map was filtered again using the constraint of the target area. The stellar map is handled through the use of dark channel denoising. Then the result is converted into a binary image. In the stellar map, the stellar and noise are marked by eight connected domains. When the target area is larger than the threshold value $\delta$, it is regarded as a stellar field. Otherwise, it is regarded as noise. Eventually, stellar map noise is almost mitigated.

\subsection{Stellar Map Centroid Positioning Combined with Centroid Trajectory Constraints}

The traditional gray-scale weighting is to use each stellar as a primitive to locate its centroid coordinates. It is not only susceptible to interference from background noise, but also can be applied to the continuous multiframe stellar map centroid positioning. The maximum energy value of each stellar field is not unique, resulting in lower centroid positioning accuracy. In this section, the continuous multiframe stellar map is processed based on the dark channel denoising. The initial position of the centroid coordinate is constrained by centroid tracking to ensure the centroid positioning accuracy. A more intuitive display is shown in Fig. 4.

First, each stellar coordinate of the maximum energy value in every image is determined, an adaptive window with the maximum pixel energy as the center is established, and the gray-scale weighting method is adopted to locate the centroid position as the initial value. Second, the centroid of the stellar in the sequence image is determined. When the maximum energy value
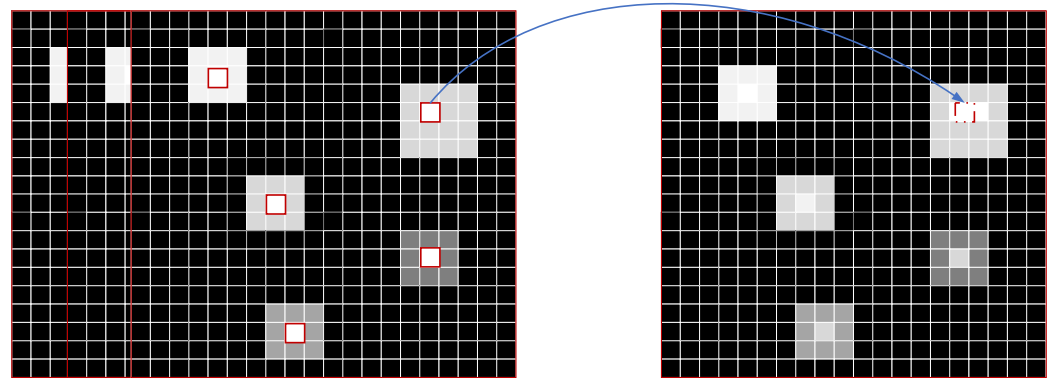

Fig. 4 Centroid positioning combined with centroid trajectory constraints. 
of a stellar exists in multiple pixels, the position of the previous stellar centroid is used to constrain the center of the adaptive window, and then the gray-scale weighting method is used to determine the stellar centroid, improving the problem that multiple maximum energies in the stellar affect the centroid positioning. The gray-scale weighting function of the step is described as

$$
\left\{\begin{array}{l}
x_{\mathrm{sub}}=\frac{\sum_{i=1}^{n} \sum_{j=1}^{n} x_{i} p^{2}\left(x_{i}, y_{j}\right)}{\sum_{i=1}^{n} \sum_{j=1}^{n} p^{2}\left(x_{i}, y_{j}\right)} \\
y_{\mathrm{sub}}=\frac{\sum_{i=1}^{n} \sum_{j=1}^{n} y_{i} p^{2}\left(x_{i}, y_{j}\right)}{\sum_{i=1}^{n} \sum_{j=1}^{n} p^{2}\left(x_{i}, y_{j}\right)}
\end{array}\right.
$$

In Eq. (2), $\left(\bar{x}_{0}, \bar{y}_{0}\right)$ is centroid coordinates, $n$ is the size of the window, $\left(x_{i}, y_{i}\right)$ is the coordinate of the $i$ 'th pixel, and $p\left(x_{i}, y_{j}\right)$ is the gray value of the $i$ 'th pixel.

\subsection{Stellar Centroid Positioning Accuracy Evaluation}

From a theoretical point of view, the diagonal distance calculated by any two stars in the navigation star catalog is equal to the diagonal distance calculated by the corresponding observation stellar coordinates, as shown in Fig. 5. The diagonal distance calculated in the navigation star catalog is regarded as the theoretical true value, and the observed diagonal distance is compared with the theoretical true value to evaluate the centroid positioning accuracy of the stellar map.

Suppose $\left(\alpha_{i}, \delta_{i}\right)$ and $\left(\alpha_{j}, \delta_{j}\right)$ are the right ascension and declination coordinates of the stars with $i$ 'th and $j$ 'th, respectively, then the mathematical description of the diagonal distance $\rho_{i j}$ between them is as follows:

$$
\rho_{i j}=\arccos \left(\left[\begin{array}{c}
\cos \alpha_{i} \cos \delta_{i} \\
\sin \alpha_{i} \cos \delta_{i} \\
\sin \delta_{i}
\end{array}\right]^{\mathrm{T}}\left[\begin{array}{c}
\cos \alpha_{j} \cos \delta_{j} \\
\sin \alpha_{j} \cos \delta_{j} \\
\sin \delta_{j}
\end{array}\right]\right)
$$

After the two stars are imaged by the star tracker, the coordinates in the stellar map are $\left(x_{i}, y_{i}\right)$ and $\left(x_{j}, y_{j}\right)$. The diagonal distance of the observed star is calculated based on the positioning result of the stellar centroid. The angular distance $\rho_{i j}^{\prime}$ can be calculated by the following equations:

$$
\begin{gathered}
w_{i}=\frac{1}{\sqrt{\left(x_{i}-x_{0}\right)^{2}+\left(y_{i}-y_{0}\right)^{2}+f^{2}}}\left(\begin{array}{c}
-\left(x_{i}-x_{0}\right) \\
-\left(y_{i}-y_{0}\right) \\
f
\end{array}\right), \\
\rho_{i j}^{\prime}=\arccos \left(w_{i}^{\mathrm{T}} w_{j}\right),
\end{gathered}
$$

where $x_{0}, y_{0}$, and $f$ are azimuth elements in the star tracker.

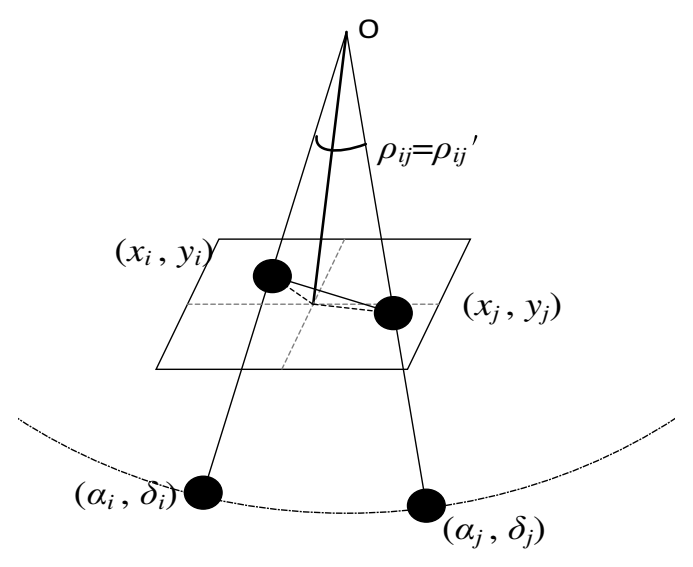

Fig. 5 Diagonal distance between navigation star and observation star. 


\subsection{Jitter Detection Based on the Continuous Multiframe Centroid Positioning}

The area array camera of star trackers on the satellite platform has a short exposure time and records all the stellar information in the field of view. Theoretically, if the orbiting satellite platform is in a stable condition, the trajectory of the stellar in the image should be a straight line in a short time. In fact, the trajectory is not linear due to jitter, which is caused by the satellite platform in orbit. The proposed centroid positioning method is used to locate the stellar centroid coordinates and the stellar coordinates are calculated in continuous multiframe stellar maps. Then the centroid trajectory is obtained. The stellar trajectory recorded in the stellar map is approximately a circular arc, so the unary quadratic equation is used to fit the trajectory. On the basis of trajectory fitting, the residual error between the true trajectory of the stellar and the fitted trajectory is calculated. According to the distribution of the residual waveform, a trigonometric function is used to fit the displacement residuals, and a fitting function model is constructed in the following equation:

$$
f(x)=a \cos (2 \pi \omega x+b)
$$

where $a$ is the amplitude, $w$ is the frequency, and $b$ is the primary phase. Based on the principle of least squares, the frequency and amplitude of attitude jitter are obtained by iterative solution. According to Eq. (6), the feasibility of centroid positioning in satellite attitude jitter detection can be tested.

\section{Experimental Results and Analysis}

\subsection{Experimental Data}

In order to verify the denoising effect of the proposed method that is applied to the ZY-3 stellar map, many orbit stellar maps acquired from the ZY-3 satellite platform are used to analyze the denoising stability and centroid positioning accuracy. In this experiment, orbital 000381 and 016891 stellar maps were randomly selected as the experimental data. Stellar maps were taken on February 3, 2012, and January 24, 2015. The size of each stellar map is 1024 pixels $\times$ 1024 pixels. The technical index of the APS star trackers on the ZY-3 satellite platform is shown in Table 1, where the stellar map exposure time is $250 \mathrm{~ms}$ and the image output frequency is $2 \mathrm{~Hz}$ by default.

Table 1 Key parameters of ZY-3 star tracker.

\begin{tabular}{ll}
\hline \hline Index & \multicolumn{1}{c}{ Parameters } \\
\hline Focal length & $43.3 \mathrm{~mm}$ \\
Principal point & 512,512 \\
Area-array image size & $1024 \times 1024$ \\
Field angle & $20 \mathrm{deg} \times 20 \mathrm{deg}$ \\
Pixel size & $15 \mu \mathrm{m} \times 15 \mu \mathrm{m}$ \\
Exposure times & $125,200,250,500 \mathrm{~ms}$ are tunable \\
Output frequency & $2 \mathrm{or} 1.66 \mathrm{~Hz}$ can be selected \\
Optical pointing precision & $5 \mathrm{arc} \mathrm{sec}(3 \sigma)$ \\
Update frequency & $4 \mathrm{~Hz}$ \\
\hline \hline
\end{tabular}




\subsection{Experimental Analysis}

In the experiment, these stellar maps were acquired from different orbits, as shown in Fig. 6. Obviously, both on the same and different orbital stellar maps, the exposures on the left are relatively strong and unstable, especially the bottom left, which presents the periodic changes. The energy value of the background noise (including dead pixels) is at least over 30, the highest is more than 90, and the bottom left corner area floats larger. Therefore, the background noise of the stellar map almost has common features based on a large number of stellar map noise information statistics. More explicit feature descriptions such as band noise, exposure intensity, and the stellar positioning changed, particularly the gray value of background noise, is much lower than that of the stellar field. Motivated by this, the background noise template based on dark channel is proposed.

In the calculation of the dark channel background noise template, the time of all stars move out of the position of the first stellar map, which is first calculated. Then the number of stellar map frames acquired by the star tracker is counted. In this paper, the number of stellar map frames $c$ is 6 . The minimum gray value of each pixel in these stellar maps is taken out and stored in a two-dimensional matrix template. This is the dark channel noise template. Orbital 000381 dark channel noise template and its three-dimensional (3D) view visualization are shown in Fig. 7.

Aiming at characterizing the noise information, the dark channel noise template is used to mitigate the stellar map obtained by APS. The system noise is well mitigated and the band noise is obviously weakened based on the dark channel noise template processing. Meanwhile, the unevenness of the center and edge regions of the stellar map is also improved. In order to further analyze the effect of the dark channel noise template on the denoising of the stellar map, the star

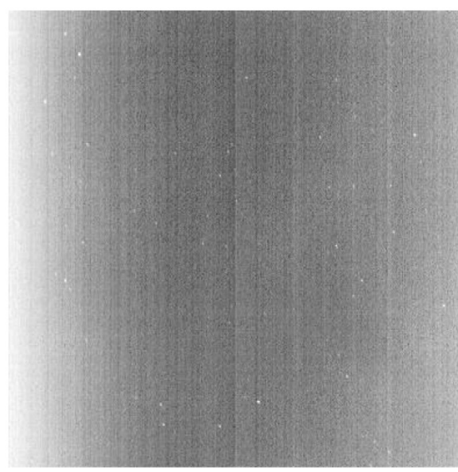

(a)

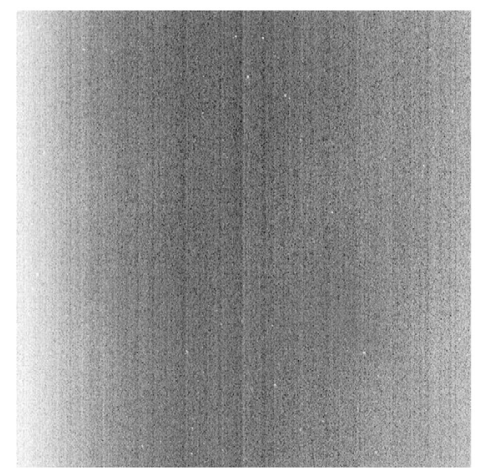

(b)

Fig. 6 Original stellar map: (a) orbital 000381 stellar map and (b) orbital 016891stellar map.
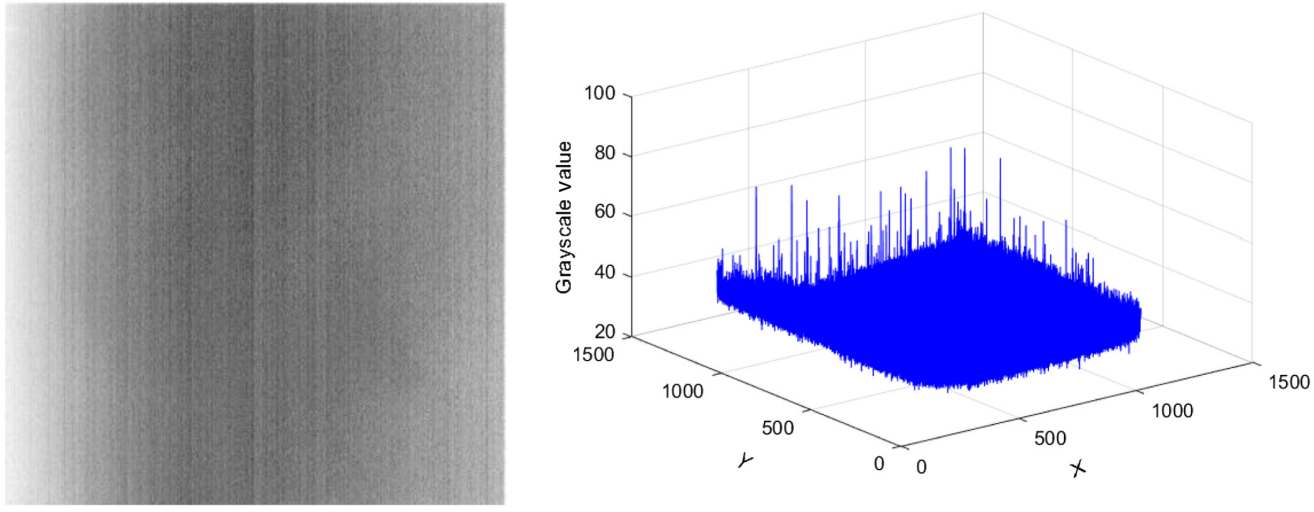

Fig. 7 Dark channel noise template and its 3D view visualization. 


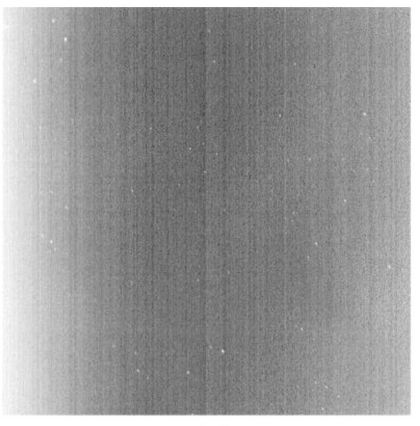

(a)

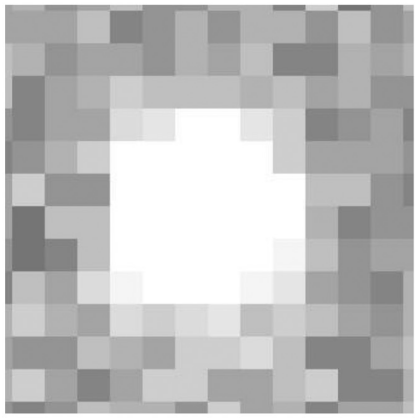

(c)

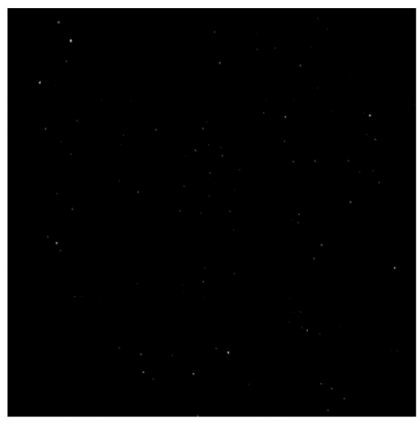

(b)

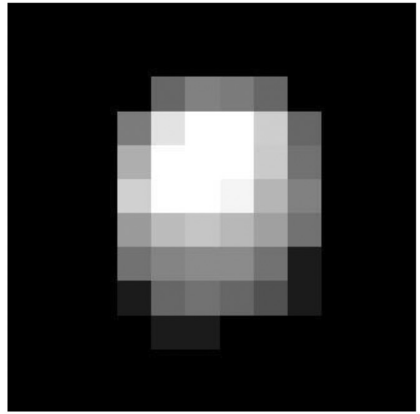

(d)

Fig. 8 Comparison of stellar map before and after noise removal: (a) before noise mitigation; (b) after noise mitigation; (c) partial zoom before noise mitigation; and (d) partial zoom after noise mitigation.

in the stellar map after denoising is partially enlarged, as shown in Fig. 8. It can be seen from the result that the radius of the spot has not changed, which ensures the reliability of the spot, improves the signal-to-noise ratio of the stellar map, and provides a guarantee for further improving the center positioning accuracy of the stellar field.

In addition, the Gaussian filtering method and the global threshold method are used to compare the effect of the noise mitigation. For example, one of the frame stellar maps is randomly selected to present. The results including the Gaussian filtering, global threshold method, and the dark channel denoising are shown in Fig. 9.

Figure 9 intuitively shows that the stellar map is denoised by the Gaussian filtering method, which smooths the whole stellar map. The noise is not removed effectively and the gray value of the star point is weakened. When the global threshold method is used to mitigate the noise, the edge band noise is still obvious and speckle noise remains. During the process of global threshold method denoising, it needs to calculate the global threshold of each frame stellar map, which has poor generalization. In contrast, the denoising method proposed in this paper can mitigate the band noise and background noise in the stellar map effectively and has good expansibility.

In order to further analyze the performance of the stellar map denoising method, centroid positioning combined with centroid trajectory constraints is adopted to evaluate and analyze the effectiveness of the stellar map denoising method. Furthermore, stellar maps acquired from the same or different orbits are used in this experiment. The star diagonal error results of the different orbits using different filtering methods are given in Fig. 10. In Fig. 10, the $X$ axis represents the number of diagonal distances between any two stars in the stellar map, and the $Y$ axis represents the error of the angular distance between any two stars in the navigation star catalog.

It can be found from different orbital stellar maps that the star diagonal error is significantly reduced based on the dark channel denoising method. This comparative experiment can indicate that the background noise is effectively mitigated and the extraction accuracy of stellar centroid is also improved. In addition, the star diagonal error is mostly concentrated near 

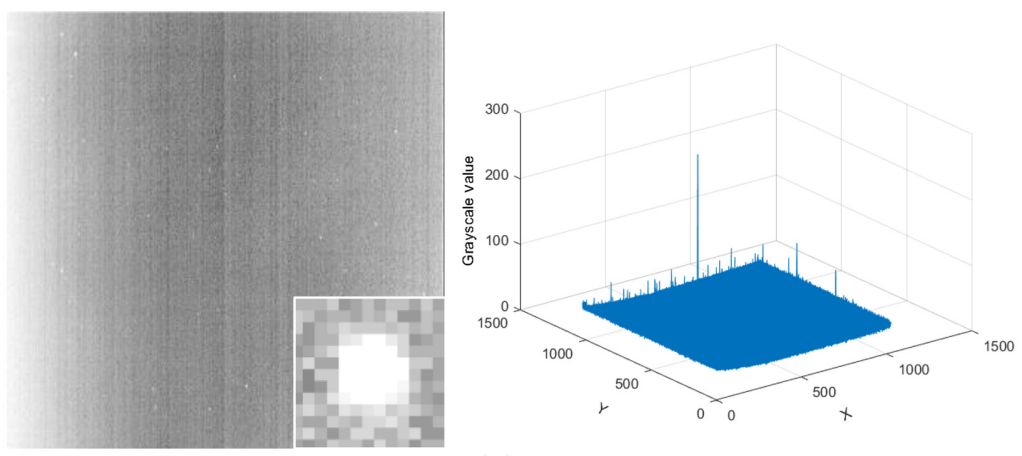

(a)
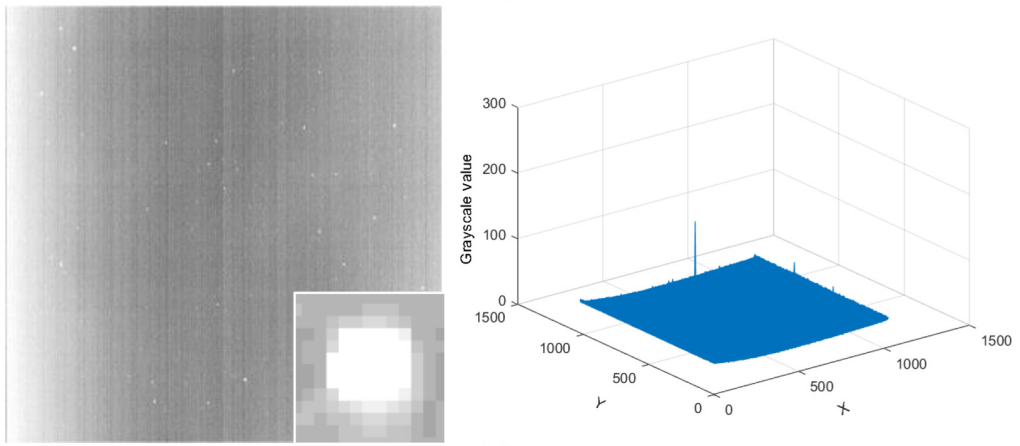

(b)
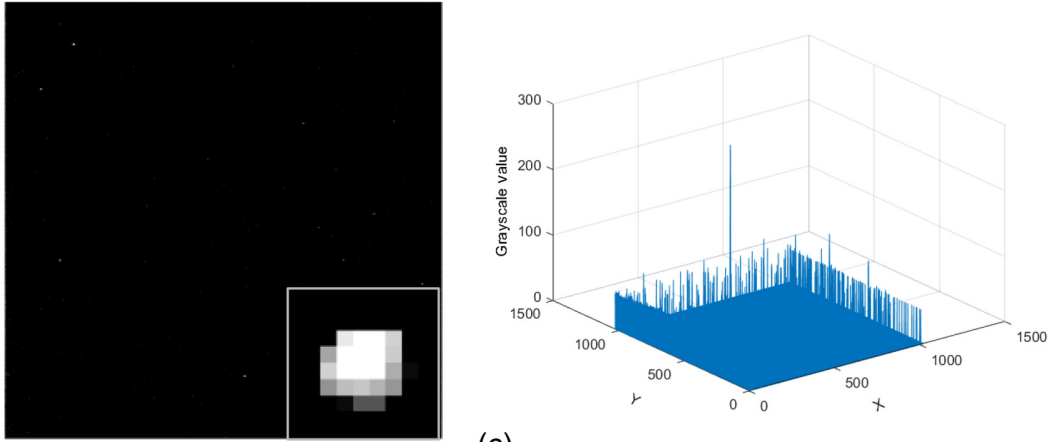

(c)
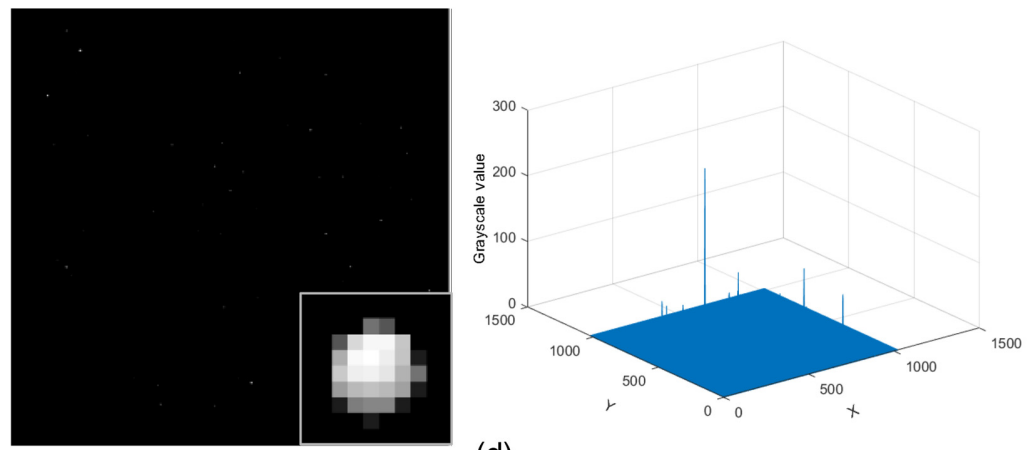

(d)

Fig. 9 Comparison of noise mitigation results based on different methods (the denoising result is on the left, the 3D view is on the right): (a) original stellar map; (b) Gaussian filtering method denoising; (c) global threshold method denoising; and (d) dark channel denoising.

0 arc sec, and the individual star diagonal error is relatively larger. There is a jump phenomenon in the star diagonal result. This jump phenomenon may be related to the exposure integration time charge transfer error, stellar spots of different magnitudes, distortion, or other factors. Orbit 000381 diagonal distance errors with time were counted. In this result, all the diagonal distances were sorted. The star angular distance results are compared in Fig. 11, 


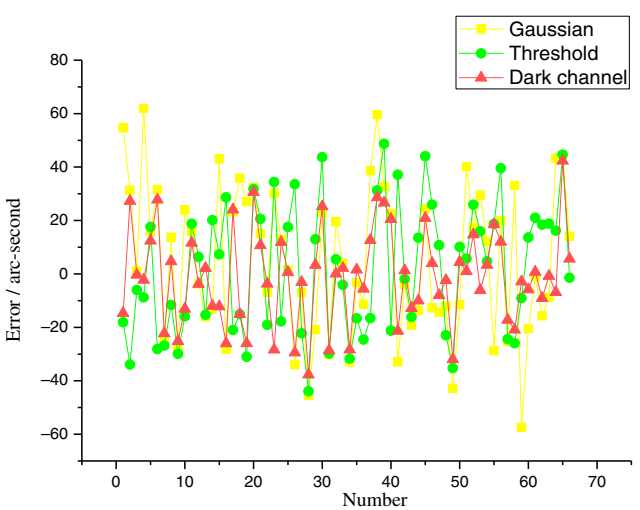

(a)

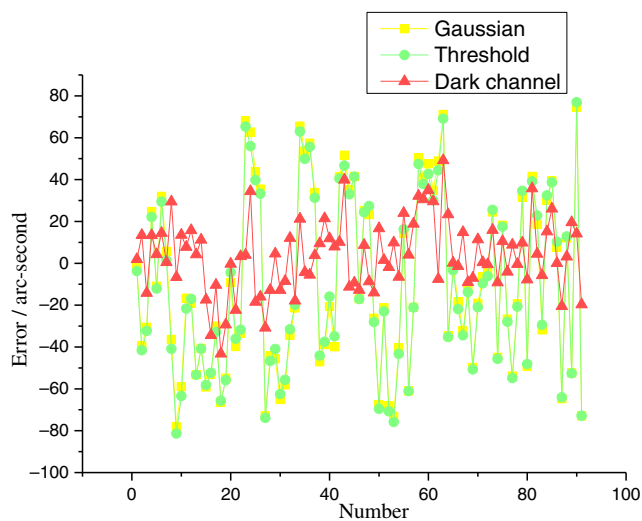

(b)

Fig. 10 Star diagonal error results of different orbit-acquired stellar maps: the result of (a) orbital 000381 and (b) orbital 016891.

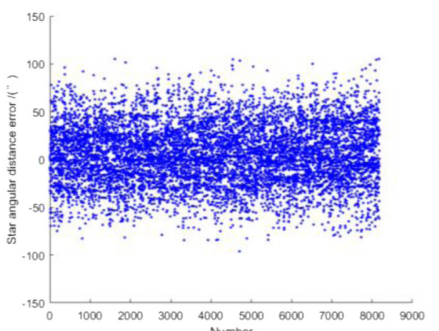

(a)

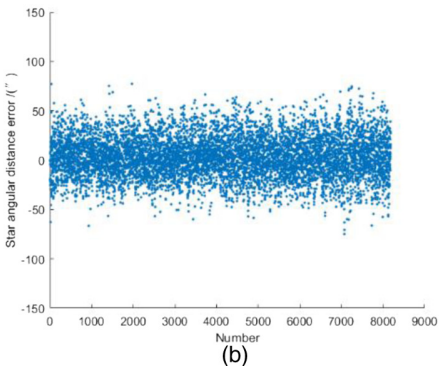

(b)

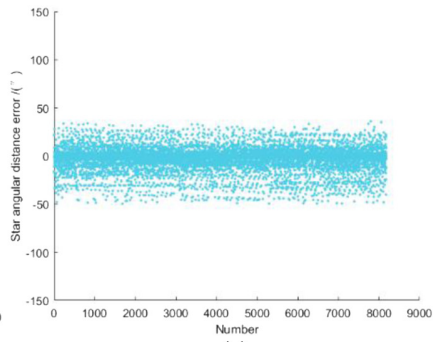

(c)

Fig. 11 Tar angular distance error results: (a) Gaussian filtering denoising; (b) global threshold denoising; and (c) proposed method.

which are handled through the use of Gaussian filtering, global threshold segmentation, and the dark channel denoising method.

As shown in Fig. 11, the distribution range of the star angular distance error based on Gaussian filtering denoising is [-96.03,105.37 arc sec]], the distribution range of the star angular distance error based on global threshold segmentation denoising is [-74.93, 77.27 arc sec], and the distribution range of the star angular distance error based on dark channel denoising is $[-49.69,39.99$ arc sec $]$. The standard deviation of the star angular distance denoised by Gaussian filtering is 31.38 arc sec, the standard deviation of the star angular distance denoised by the global threshold segmentation method is $20.56 \mathrm{arc} \mathrm{sec}$, and the standard deviation of the star angular distance with dark channel denoising is $12.53 \mathrm{arc} \mathrm{sec}$. The range of the star angular distance error floating range of the proposed method is obviously better than those of the Gaussian filtering and global threshold denoising methods; evidently, the method proposed in this paper can improve the extraction accuracy of the stellar map centroid and further improve the positioning accuracy of the star tracker.

The method proposed in this paper can estimate the background noise of the stellar map according to the external environment and the changes of the response device, so that the denoising method has good timeliness and universality. At present, it has been fully applied in the ZY-3 application system and can be popularized and applied in other satellite stellar map preprocessing in the future. Meanwhile, the change of the stellar centroid positioning with higher brightness is counted in the experiment, and the jitter frequency of the satellite platform is analyzed. The changes in the centroid positioning of two stars are shown in Fig. 12.

In Fig. 12, it can be roughly found that the stellar centroid has changed and there is a certain regularity. The motion trajectories in the $X$ and $Y$ directions of the stellar centroid are fitted by the unary quadratic equation and the fitting residuals are calculated. The least squares method is used to fit the data residuals. The fitting function can be determined by prior knowledge and the 
Zhu et al.: Stellar map centroid positioning based on dark channel denoising and feasibility...

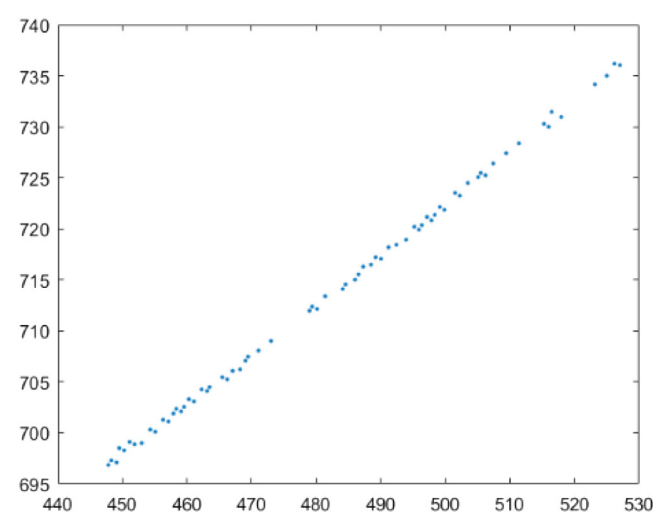

(a)

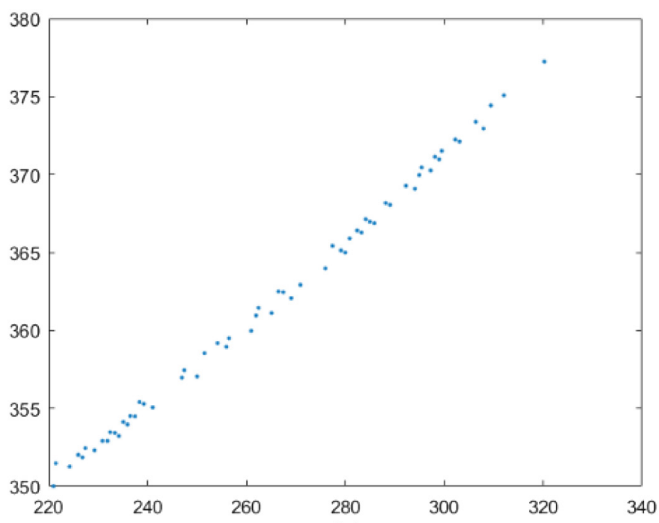

(b)

Fig. 12 Stellar centroid trajectory: (a) 6th stellar and (b) 10th stellar.

Table 2 Stellar modeling results and accuracy.

\begin{tabular}{lcc}
\hline \hline 6th stellar & $x$ direction & $Y$ direction \\
\hline & $f(x)=a * \cos (w * x+b)$ & $f(x)=a * \cos (w * x+b)$ \\
& Coefficients: & Coefficients: \\
& $a=2.381$ & $a=1.122$ \\
& $w=0.67$ & $w=0.67$ \\
Modeling accuracy (pixel) & $b=1.153$ & $b=0.9951$ \\
10th stellar & 1.36 & 0.57 \\
& $a * \cos (w * x+b)$ & $f(x)=a * \cos (w * x+b)$ \\
& Coefficients: & Coefficients: \\
Modeling accuracy (pixel) & $a=2.611$ & $a=0.5952$ \\
\hline \hline
\end{tabular}

undetermined coefficients in the fitting function are determined by the least square method. However, the real jitter state of the satellite platform is unknown. In order to evaluate the fitting effect more objectively, the sum of the square due to error is used to analyze the fitting result in the experiment. In this evaluation method, the smaller the sum of the square due to error is, the better the fitting results are. Fast Fourier transform is used to analyse the residual and the frequency, amplitude, initial phase, and initial value of constant of the residual curve can be obtained, respectively, as shown in Table 2.

The ZY-3 satellite platform was detected to have a jitter frequency of $0.67 \mathrm{~Hz}$. The amplitude in the $X$ direction is about 2 pixels and the amplitude in the $Y$ direction is about 1 pixel, as shown in Fig. 13. This jitter frequency has a relatively small effect on the intersection error of the stereoscopic image compared to the 2.1-m resolution remote-sensing image acquired by ZY-3, and therefore, has not been compensated in actual production. Jitter detection based on the stellar map centroid change can be realized without any interference from the meteorological conditions. The results of jitter detection based on the stellar map are basically consistent with the results based on a multispectral image. This also verifies that the existence of jitter in the ZY-3 platform with a frequency range of 0.6 to $0.7 \mathrm{~Hz}$. 


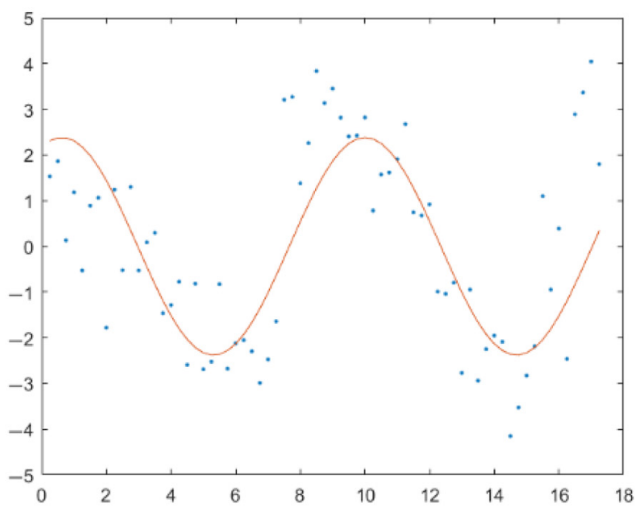

(a)

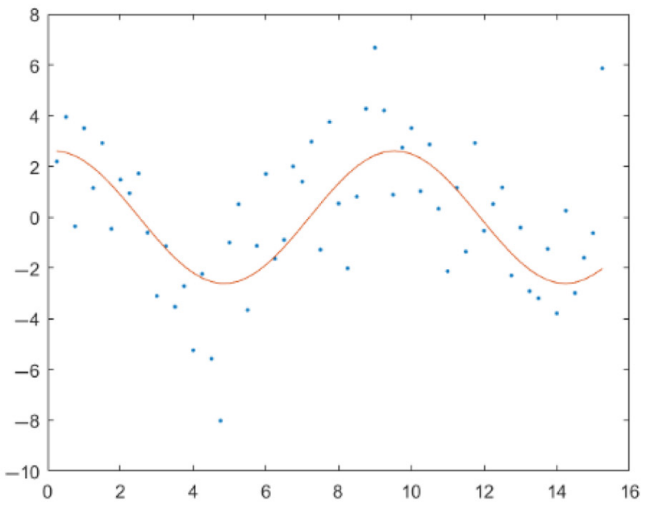

(c)

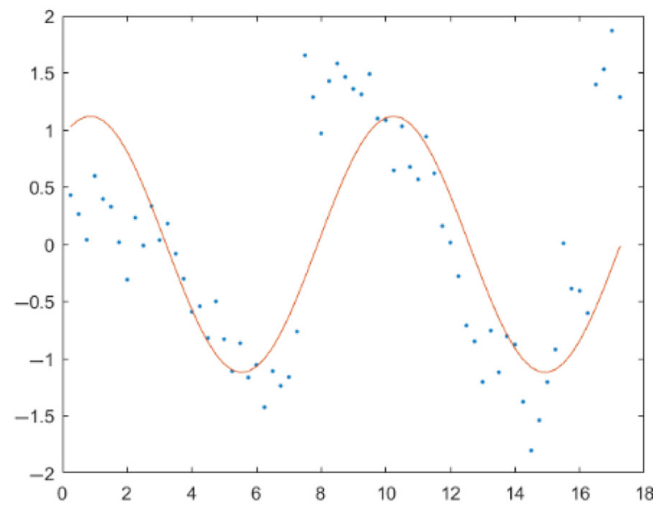

(b)

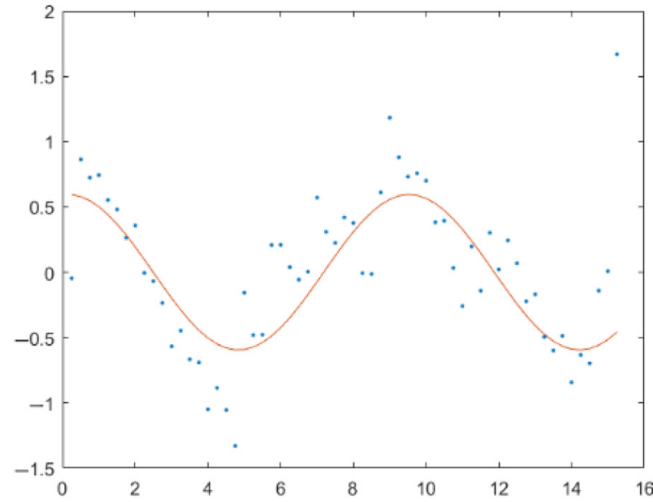

(d)

Fig. 13 Residual fitting results of stellar centroid positioning changes: (a) $X$ residual fitting of the 6th stellar; (b) $Y$ residual fitting of the 6th stellar; (c) $X$ residual fitting of the 10th stellar; and (d) $Y$ residual fitting of the 10th stellar.

\section{Conclusion}

In this paper, the jitter frequency of a satellite platform based on continuous multiframe centroid positioning results is proposed. The content mainly includes stellar map denoising based on dark channel and the continuous multiframe stellar map centroid positioning combined with centroid trajectory constraints. The experiment uses stellar maps of ZY-3 as an example, and the denoising effect and positioning accuracy of the stellar centroid are increased. It has laid a certain foundation for improving the sensitivity of satellite platform jitter detection. The specific conclusions are as follows.

1. By comparing the results of the stellar map and $3 \mathrm{D}$ visualization results based on different denoising methods, the dark channel denoising method clearly and effectively solves the problem of stripe noise and background noise in stellar maps. Compared with the Gaussian filtering denoising method, the star angular distance error is improved by 18.85 arc sec, and compared with the global threshold segmentation method, the star angular distance error is improved by 8.03 arc sec. The experimental results show that the proposed method can improve the accuracy of centroid positioning and provide technical support for the postpositioning accuracy of star trackers.

2. In this paper, the feasibility of jitter detection of the ZY-3 satellite platform based on the centroid change in the continuous multiframe stellar map is the first to be analyzed. A jitter frequency of $0.67 \mathrm{~Hz}$ from the ZY-3 satellite platform was detected. The amplitude in the $X$ direction is about 2 pixels, and the amplitude in the $Y$ direction is about 1 pixel. This has little effect on the intersection error of the ZY-3 satellite image. Therefore, it is not necessary to compensate for jitter in actual production. 
In the future, satellite sensors and the agility of the satellite platform will be improved. The influence of jitter on the geometric accuracy and mapping accuracy of satellite images will become increasingly significant. An integrated multiple jitter detection method can be developed, providing strong technical support for the stability of the satellite platform.

\section{Acknowledgments}

This study was financially supported in part by the Hebei Province Science and Technology Research Project (Grant No. Z2020119); the Fundamental Research Funds for the Central Universities (Grant No. ZY20200202); National Key Research and Development Project of China (Grant No. 2016YFB0501005); High Remote Sensing, Surveying, and Mapping Application Demonstration System (Project No. AH1601-8); the National Natural Science Foundation of China (Project Nos. 41301525, 41571440, 61601213, 41601505, 41871379, and 41871382); the Special Scientific Research Fund of Public Welfare Surveying and Mapping Profession of China (Project Nos. 201412007 and 201512012); National Key Research and Development Program (Project No. 2017YFB0504201); the project funded by China Postdoctoral Science Foundation (Project Nos. 2017M611252); the Project of Liaoning Education Department (Grant Nos. 16-1096 and LR2016045); Public Welfare Research Fund in Liaoning Province (Grant No. 20170003); National Key R \& D Program for Strategic International Scientific and Technological Innovation Cooperation of China (Grant No. 2016YFE0205300); National Key Research And Development Project of China (Grant No. 2016YFB0501005); and Active and Passive Composite Mapping and Application Technology with Visible, Infrared, and Laser Sensors (Grant No. D040106), in part by the Research and Development Forest Resource Dynamic Monitoring and Forest Volume Estimation using LiDAR remote sensing (2020YFE0200800), in part by the High Resolution Remote Sensing, surveying and mapping Application Program (No. 1), and Multi Beam Laser Terrain Detection Radar and its Application Technology D040105, in part by Authenticity Validation Technology of Elevation Accuracy of GF-7 Laser Altimeter under Grant 42-Y20A11-9001-17/18, Surveying and Mapping Application Demonstration Project of GaoFen-2 (42-Y30B04-9001-19/21), the Seed fund for Jinqiao project of Beijing Association for Science and Technology Grant ZZ19013, the key R\&D plan of the Ministry of Science and Technology (2017YFB0504201), and in part by the Research on satellite observation system and application scheme for fine and fast stereo monitoring of land space.

\section{References}

1. J. Lu, Y. Yang, and H. Zhang, "A hybrid method for accurate star tracking using star sensor and gyros," Rev. Sci. Instrum. 88(10), 105004 (2017).

2. G. Mcvittie, Development and Performance Characterization of Colour Star Trackers. Ryerson University (2013).

3. K. Ho, "A survey of algorithms for star identification with low-cost star trackers," Acta Astron. 73, 156-163 (2012).

4. X. Fei et al., "A novel approach based on MEMS-Gyro's data deep coupling for determining the centroid of star spot," Math. Prob. Eng. 2012(6), 55-59 (2012).

5. V. Schiattarella, D. Spiller, and F. Curti, "A novel star identification technique robust to high presence of false objects: the multi-poles algorithm," Adv. Space Res. 59(8), 2133-2147 (2017).

6. W. Zhang, W. Quan, and L. Guo, "Blurred star image processing for star sensors under dynamic conditions," Sensors 12(5), 6712-6726 (2012).

7. M. Wang, J. Y. Zhao, and T. Chen, "Center extraction method for star-map targets based on anisotropic Gaussian surface fitting," Acta Opt. Sin. 37(5), 218-227 (2017).

8. M. Zhou, Y. Shi, and J. Yang, "Denoising star map data via sparse representation and dictionary learning," Optik 126(11-12), 1133-1137 (2015).

9. J. Jiang et al., "Rapid star tracking algorithm for star sensor," IEEE Aerosp. Electron. Syst. Mag. 24(9), 23-33 (2009). 
Zhu et al.: Stellar map centroid positioning based on dark channel denoising and feasibility...

10. X. Tang et al., "Overview of Earth observation satellite platform microvibration detection methods," Sensors 20(3), 736 (2020).

11. M. Wang et al., "Extremum median filter map denoising algorithm based on energy function,” J. Electron. Inf. Technol. 39(6), 1387-1393 (2017).

12. Q. Quan et al., "Star map processing algorithm of star sensor and autonomous celestial navigation," in INS/CNS/GNSS Integrated Navigation Technology, Springer, Berlin, Heidelberg (2015).

13. A. B. Katake, Modeling, Image Processing and Attitude Estimation of High Speed Star Sensors, Texas A\&M University, Texas, pp. 103-104 (2009).

14. B. Jahne, Practical Handbook on Image Processing for Scientific Application, 2nd ed., CRC Press, Boca Raton, Florida (2004).

15. H. Wang et al., "Gaussian analytic centroiding method of star image of star tracker," $A d v$. Space Res. 56(10), 2196-2205 (2015).

16. X. Feng, "Fast algorithms on center location of two dimensional Gaussian distribution spot," Acta Opt. Sin. 32(5), 0512002 (2012).

17. X. Yuan et al., "Centroid automatic extraction of spaceborne laser spot image," Acta Geod. Cartogr. Sin. 47(2), 135-141 (2018).

18. X. Tang et al., "ZY3-02 laser altimeter on-orbit geometrical calibration and test," Acta Geod. Cartogr. Sin. 46(6), 714-723 (2017).

19. F. Mo et al., "An attitude modelling method based on the inherent frequency of a satellite platform," ISPRS-Int. Arch. Photogramm. Remote Sens. Spatial Inf. Sci. XLII-1/W1, 29-33 (2017).

20. X. Tang et al., "The China ZY3-03 Mission: surveying and mapping technology for highresolution remote sensing satellites," IEEE Geosci. Remote Sens. Mag. 8(3), 8-17 (2019).

Hong Zhu received her PhD in photogrammetry and remote sensing from Liaoning Technical University, Huludao, China, in 2017. She is currently working as an associate professor at the Institute of Disaster Prevention (CIDP). Her research includes image matching, remote sensing image super resolution reconstruction, and satellite attitude determination.

Junfeng Xie received his $\mathrm{BS}$ degree and $\mathrm{PhD}$ in remote sensing information engineering from Wuhan University, China, in 2004 and 2009, respectively. He is currently working as a researcher at the Land Satellite Remote Sensing Application Center (LASAC) of Ministry of Natural Resources of China. His research includes satellite attitude determination, spaceborne laser altimeter calibration, and remote sensing application.

Xinming Tang received his MSc degree in land administration in 1998 and his PhD in geoinformation science and computer application in 2004 both from the University of Twente, Enschede, The Netherlands. He is currently the academic deputy director of the LASAC, MNR, Beijing, China. His research interests focus on spatial information science and technology, including remote sensing, geographic information systems, and their integration.

Di Jia received his PhD in computer application from Northeastern University. He is a professor at Liaoning Technical University, Huludao, China. His main research interests include stereo matching and 3D reconstruction, and computer vision positioning system, etc.

Guangtong Sun received his MA degree in photogrammetry and remote sensing from Liaoning Technical University, Huludao, China, in 2011. He is currently working as an associate professor at the CIDP. He is mainly engaged in disaster remote sensing monitoring theory and application research. 